\title{
Rab23 contributes to the progression of colorectal cancer via protein kinase $B$ and extracellular signal-regulated kinase signaling pathways
}

\author{
TONGBI ZHAO* , DONG HAN* and HUAN MENG \\ Digestive Department, Shanxian Central Hospital of Shandong Province, Heze, Shandong 274300, P.R. China
}

Received June 8, 2018; Accepted April 10, 2019

DOI: $10.3892 / \mathrm{ol} .2019 .10491$

\begin{abstract}
The role of Ras-related protein Rab23 in tumors has attracted increasing attention in recent years; however, whether it can function as an oncogenic protein remains under debate, and its role in colorectal cancer (CRC) is currently unknown. In the present study, high expression of Rab23 in CRC tissues was confirmed using immunohistochemistry, and high expression of Rab23 in CRC cells (SW1116 and HT29) was confirmed using reverse transcription-polymerase chain reaction and western blot analysis. A positive association of Rab23 with tumor size and advanced clinical stage was confirmed by $\chi^{2}$ analysis. In addition, the positive association of Rab23 with poor disease-free survival was confirmed by survival analysis. Cell experiments further demonstrated that overexpression of Rab23 increased the expression of the proliferation marker $\mathrm{Ki}-67$ and the proliferative ability in SW1116 and HT29 cells. Molecular mechanism research revealed that the extracellular signal-regulated kinase (ERK) and protein kinase $\mathrm{B}$ (AKT) signaling pathways contributed to the high expression of $\mathrm{Ki}-67$ and increased the proliferative ability induced by Rab23 in CRC cells. In conclusion, the study confirmed the high expression of Rab23 in CRC, and its positive association with CRC progression and poor prognosis. Furthermore, the data demonstrated that Rab23 increased the proliferation of CRC cells via the ERK and AKT signaling pathways. These results suggest that Rab23 may be used as a protein for diagnosis and prognosis prediction in patients with CRC, and is proposed to be a novel therapeutic target for improving the patient outcome.
\end{abstract}

Correspondence to: $\mathrm{Dr}$ Huan Meng, Digestive Department, Shanxian Central Hospital of Shandong Province, 1 Wenhua Road, Shanxian, Heze, Shandong 274300, P.R. China

E-mail: menghuanathe@163.com

\section{*Contributed equally}

Key words: Rab23, colorectal cancer, proliferation, overall survival, disease-free survival

\section{Introduction}

Colorectal cancer (CRC) is one of the most common malignant tumors and the fourth leading cause of cancer-associated mortality worldwide (1). In recent years, despite improvements in precancerous screening (2-4), surgical resection (1), chemotherapy (5), radiotherapy (6) and target therapy (5), patients with CRC still exhibit poor prognosis, particularly patients at advanced stages of the disease (7). Therefore, it is crucial to identify novel therapeutic targets to improve the prognosis of CRC patients.

Ras-related protein Rab23, a member of the Ras-related small GTPase family, was first isolated from the brain in 1994 (8). Studies from the past three decades have demonstrated that Rab23 serves an important role in endosomal membrane trafficking $(9,10)$. A further molecular mechanism study revealed that it may negatively regulate Sonic hedgehog (Shh) signaling pathway in an indirect manner (11). In recent years, the role of Rab23 in tumors has attracted increasing attention. In 2016, Jian et al (12) reported that Rab23 promoted cutaneous squamous cell carcinoma migration and invasion via the integrin $\beta 1 /$ Ras-related protein Rac1 pathway. In 2018, Zhang et al (13) demonstrated that Rab23 promoted the cisplatin resistance of ovarian cancer via the Shh-Gli-ATP-binding cassette sub-family G member 2 signaling pathway. However, the role of Rab23 in CRC currently remains unknown.

In the present study, it was initially confirmed that there is a high expression of Rab23 in CRC. Subsequently, it was observed that high expression of Rab23 was positively associated with tumor progression and poor prognosis. The role of Rab23 in the proliferation of CRC cells and the potential molecular mechanism were then identified.

\section{Materials and methods}

Cell culture. Human colon epithelial FHC cells, and the human CRC cell lines SW1116 and HT29 were all purchased from the American Type Culture Collection (Manassas, VA, USA). SW1116 and HT29 cell lines were cultured in Dulbecco's modified Eagle's medium (DMEM) supplemented with $10 \%$ fetal bovine serum (FBS; both from Invitrogen; Thermo Fisher Scientific, Inc., Waltham, MA, USA) $(11,12)$. The FHC cell line was cultured in mixed medium including DMEM and 
Ham's F12 nutrient mixture, supplemented with 10\% FBS. All cells were cultured at $37^{\circ} \mathrm{C}$ in $5 \% \mathrm{CO}_{2}$.

Tissue specimen collection. Paraffin-embedded tumor tissues and clinicopathological features were obtained from $90 \mathrm{CRC}$ patients who had undergone surgery at Shanxian Central Hospital (Heze, China) between January 2008 and January 2010. Clinical stage was determined according to the American Joint Committee on Cancer system (14). The survival data were collected, and the survival time ranged between 19 and 90 months, with the median survival time of 90 months. The study was approved by the Review Board of Shanxian Central Hospital. The specimens from patients who were diagnosed with CRC by pathologists were included in the present study; patients who had received preoperative chemotherapy or irradiation were excluded.

Immunohistochemical (IHC) analysis. Tissue samples were sectioned into $4-\mu \mathrm{m}$ slices, deparaffinized in xylene, rehydrated in graded ethanol and boiled in $10 \mathrm{mmol} / \mathrm{l}$ citrate buffer (pH 6.0) for $3 \mathrm{~min}$ at $100^{\circ} \mathrm{C}$ for antigen retrieval. The expression of Rab23 and $\mathrm{Ki}-67$ in CRC tissue sections were determined using IHC staining with the following primary antibodies: Rab23 (1:400; cat. no. ab230200; Abcam, Cambridge, MA, USA) and Ki-67 (1:600; cat. no. ab833; Abcam). Subsequent to staining overnight at $4^{\circ} \mathrm{C}$ using primary antibodies, the sections were stained for $30 \mathrm{~min}$ at room temperature with secondary antibody (cat. no. 9902; Fuzhou Maixin Biotech Co., Ltd., Fuzhou, China). Assessment of IHC staining was then performed by two independent pathologists simultaneously. The estimated fraction of positively stained tumor cells was represented by the proportion score, as follows: $0, \leq 25 \%$ staining; 1, 26-50\% staining; 2, 51-75\% staining; and 3, >75\% staining. The estimated average staining intensity of positive tumor cells was represented by the intensity score, which was assigned as follows: 0 , negative; 1 , weak; 2 , moderate; and 3 , strong. The expression level of Ki-67 was evaluated using the proportion score directly, while the expression level of Rab23 was evaluated using the sum of the proportion and intensity scores, with a score of $<4$ indicating low expression and $\geq 4$ indicating high expression.

Reverse transcription-polymerase chain reaction (RT-PCR). TRIzol reagent (Invitrogen; Thermo Fisher Scientific, Inc.) was used to extract total RNA from the samples, according to the manufacturer's protocol. The RNA concentration was measured using a 2000/2000C Ultraviolet spectrophotometer (Thermo Fisher Scientific, Inc.) cDNA was synthesized using the PrimeScript RT-PCR kit (Takara Biotechnology Co., Ltd., Dalian, China) according to the manufacturer's protocol. The PCR conditions were as follows: $25^{\circ} \mathrm{C}$ for $10 \mathrm{~min}, 42^{\circ} \mathrm{C}$ for $60 \mathrm{~min}$ and then $70^{\circ} \mathrm{C}$ for $5 \mathrm{~min}$. The primers used in this study were as follows: Rab23, 5'-AGGCACTGGCAAAAAGGT YA-3' (forward) and 5'-CGGAGTGACTTCCACCAGAT-3' (reverse); GAPDH, 5'-AGAAGGCTGGGGCTCATTTG-3' (forward) and 5'-AGGGGCCATCCACAGTCTTC-3' (reverse). GAPDH was used as an internal control.

Western blot analysis. Protein was extracted from the cells using radioimmunoprecipitation assay lysis buffer containing
$1 \%$ phenylmethane sulfonyl fluoride (Beyotime Institute of Biotechnology, Haimen, China), and protein concentration was measured using the BCA kit (Pierce; Thermo Fisher Scientific, Inc.) according to the manufacturer's protocol. Next, $200 \mu \mathrm{g}$ protein was loaded in each well of 5\% acrylamide gel, separated by $10 \%$ SDS-PAGE and transferred to a nitrocellulose membrane. Subsequent to blocking in 5\% non-fat milk (cat. no. 232100; Becton, Dickinson and Company, New Jersey, USA) at room temperature for $1 \mathrm{~h}$, the membrane was probed at $4^{\circ} \mathrm{C}$ overnight using the following primary antibodies: ERK (cat. no. AF1576; 1:1,000), phosphorylated (p)-ERK (cat. no. AF1018; 1:1,000), AKT (cat. no. AF2055; 1:1,000) and p-AKT (cat. no. AF887; 1:1,000), purchased from Cell Signaling Technology, Inc. (Danvers, MA, USA); Rab23 (cat. no. ab230200; 1:300), Ki-67 (cat. no. ab833; 1:300) and GAPDH (cat. no. ab9485; 1:4,000), all purchased from Abcam (Cambridge, MA, USA). This was followed by incubation with peroxidase-linked goat anti-rabbit-IgG antibody (cat. no. ab150077; 1:4,000; Abcam) at room temperature for $1.5 \mathrm{~h}$. Signals on the membrane were detected using ECL reagents (Pierce; Thermo Fisher Scientific, Inc., Waltham, MA, USA). The results were quantified using Image-Pro software (version 5.1; Media Cybernetics, Inc., Rockville, MA, USA) (15).

Plasmid transfection. pcDNA3.1/Rab23 or control empty vector plasmid (Shanghai GenePharma Co., Ltd., Shanghai, China) were transfected into SW1116 and HT29 cells to increase the expression of Rab23. The transfection was conducted using Lipofectamine ${ }^{\circledR} 2000$ (Invitrogen; Thermo Fisher Scientific, Inc., Waltham, MA, USA) according to the manufacturer's protocol. The transfection efficiency was verified using RT-PCR and western blot analyses.

MTT assay. CRC cells were plated in a 96-well plate at a density of $3 \times 10^{3}$ cells/well. After $24,48,72,96$ and $120 \mathrm{~h}$ of incubation, the cell viability was measured by MTT assay at a wavelength of $490 \mathrm{~nm}$, according to the manufacturer's protocol (Beijing Solarbio Science \& Technology Co., Ltd., Beijing, China). Growth curves were then constructed.

AKT and ERK signaling pathway inhibition. ERK inhibitor U0126 (cat. no. S1102; Selleck Chemicals, Houston, Texas, USA) or AKT inhibitor LY294002 (cat. no. S1105; Selleck Chemicals) was added to the medium of CRC cells overexpressing Rab23 at a final concentration of $10 \mu \mathrm{M} / 1$ or $50 \mu \mathrm{M} / 1$ respectively. Cell were collected at $72 \mathrm{~h}$, and western blotting and MTT assay were performed as described above.

Statistical analysis. SPSS software version 13.0 (SPSS, Inc., Chicago, IL, USA) was used for statistical analysis. All data are expressed as the mean \pm standard deviation. Differences between three groups were analyzed using one-way analysis of variance and Dunnett's post-hoc test, while differences between two groups were analyzed using t-test. The correlation of Rab23 with clinical parameters was analyzed using $\chi^{2}$ analysis. Survival curves were constructed using the Kaplan-Meier method and compared using the log-rank test. $\mathrm{P}<0.05$ was considered to denote differences that were statistically significant. 

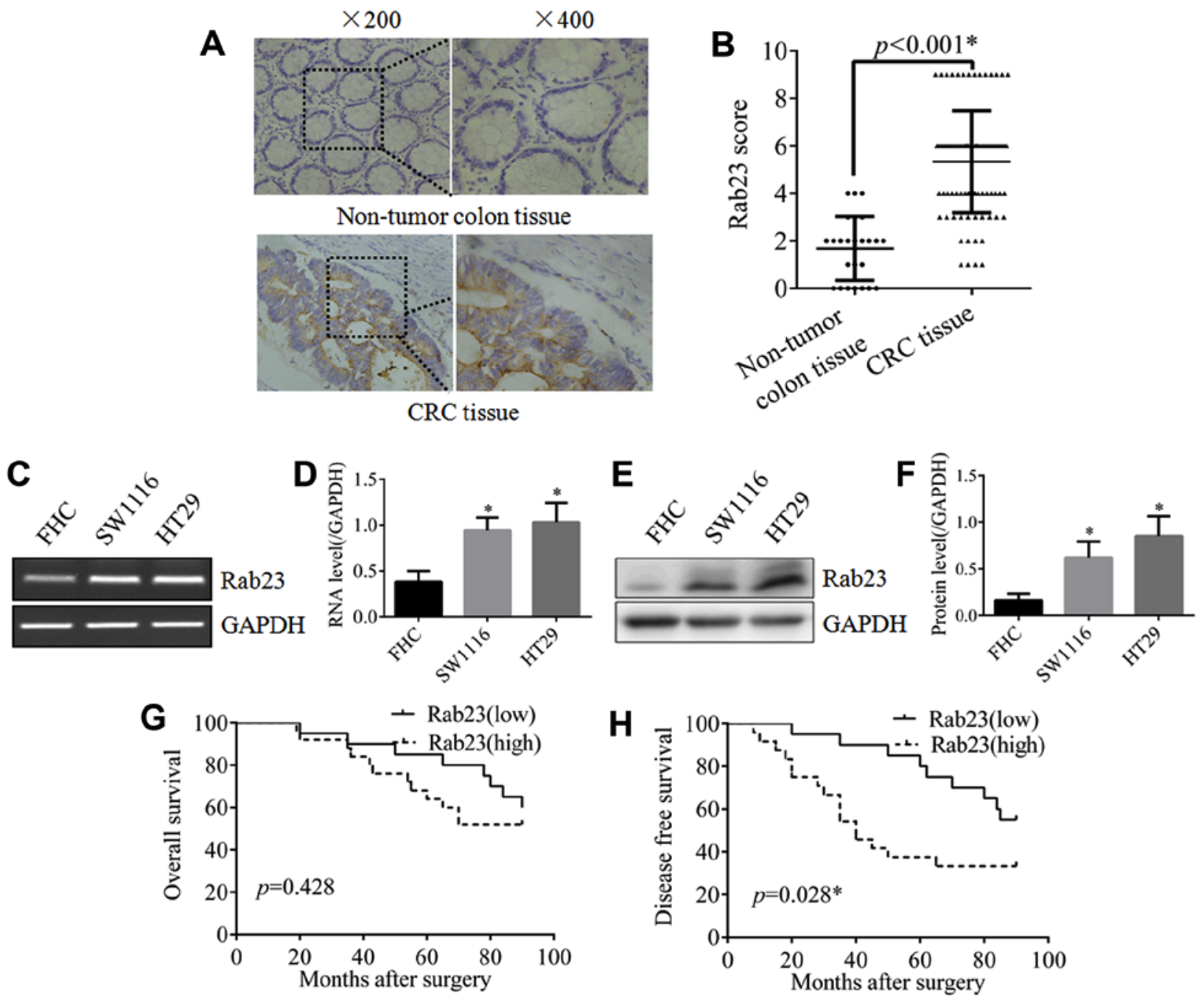

Figure 1. Rab23 is highly expressed in CRC and associated with poor prognosis. (A) Immunohistochemical staining images and (B) scores for Rab23 expression, indicating significantly stronger expression in CRC tissues compared with non-tumor colon tissues. (C) Reverse transcription-polymerase chain reaction gel and (D) quantified mRNA expression of Rab23, and (E) western blot and (F) quantified protein expression of Rab23. Higher Rab23 expression was observed in CRC cell lines SW1116 and HT29 compared with the human colon epithelial cell line FHC at the mRNA and protein levels. (G) Overall survival and (H) disease-free survival were poorer in the high Rab23 expression group in comparison with low Rab23 expression group, but with a significant difference only observed in disease-free survival. ${ }^{*} \mathrm{P}<0.05$ vs. FHC cells. CRC, colorectal cancer.

\section{Results}

Rab23 is highly expressed in CRC tissues and cells. As shown in Fig. 1A, strong staining of Rab23 was detected in CRC tissues, whereas almost no staining of Rab23 was observed in the non-tumor colon tissues. The difference between CRC and non-tumor tissues was statistically significant (Fig. 1B). To further verify the expression of Rab23 in CRC, RT-PCR and western blot analyses were performed in the CRC cell lines SW1116 and HT29. The results revealed that Rab23 expression in the two CRC cell lines was significantly higher compared with that in the human colon epithelial cell line FHC, both at the mRNA (Fig. 1C and D) and protein (Fig. 1E and F) levels. These data demonstrated that there was a high expression of Rab23 in CRC.

Rab23 is positively associated with tumor size, advanced clinical stage and poor disease-free survival (DFS). As shown in Table I, a tumor diameter of $>4 \mathrm{~cm}$ was only observed in
16 out of 36 cases in the low Rab23 expression group, which was significantly reduced in comparison with the number of cases with this diameter in the high Rab23 expression group (38 out of 54 cases). In addition, in the low Rab23 expression group, there were only 10 out of 36 cases at stages III/IV of the disease, which was significantly less than the cases at this stage in the high Rab23 expression group (29 out of 54 cases). However, there was no significant difference in terms of age, sex, differentiation degree and lymph node metastasis between the two groups.

Subsequently, in order to investigate the effect of Rab23 on prognosis, survival analysis was performed. Although the difference in overall survival (OS) between the two groups was not statistically significant, the OS of the high Rab23 expression group was poorer compared with that in the low Rab23 expression group (Fig. 1G). In addition, the DFS of the high Rab23 expression group was significantly poorer compared with that of the low Rab23 expression group (Fig. 1H). These 
Table I. Association of Rab23 expression with the clinicopathological parameters of patients with colorectal cancer.

\begin{tabular}{|c|c|c|c|c|c|}
\hline \multirow[b]{2}{*}{ Clinical parameters } & \multirow[b]{2}{*}{ Cases $(n=90)$} & \multicolumn{2}{|c|}{ Rab23 expression } & \multirow[b]{2}{*}{$\chi^{2}$} & \multirow[b]{2}{*}{ P-value } \\
\hline & & Low $(\mathrm{n}=36)$ & High $(\mathrm{n}=54)$ & & \\
\hline \multicolumn{6}{|l|}{ Age (years) } \\
\hline$\leq 65$ & 40 & 17 & 23 & \multirow[t]{2}{*}{0.188} & \multirow[t]{2}{*}{0.665} \\
\hline$>65$ & 50 & 19 & 31 & & \\
\hline \multicolumn{6}{|l|}{ Sex } \\
\hline Male & 54 & 25 & 29 & \multirow[t]{2}{*}{2.23} & \multirow[t]{2}{*}{0.135} \\
\hline Female & 36 & 11 & 25 & & \\
\hline \multicolumn{6}{|l|}{ Tumor size } \\
\hline$\leq 4 \mathrm{~cm}$ & 36 & 20 & 16 & \multirow[t]{2}{*}{6.049} & \multirow[t]{2}{*}{$0.014^{\mathrm{a}}$} \\
\hline$>4 \mathrm{~cm}$ & 54 & 16 & 38 & & \\
\hline \multicolumn{6}{|l|}{ Differentiation } \\
\hline Low & 36 & 14 & 22 & \multirow[t]{2}{*}{0.031} & \multirow[t]{2}{*}{0.861} \\
\hline High/moderate & 54 & 22 & 32 & & \\
\hline \multicolumn{6}{|l|}{ Clinical stage } \\
\hline $\mathrm{I} / \mathrm{II}$ & 51 & 26 & 25 & \multirow[t]{2}{*}{5.913} & \multirow[t]{2}{*}{$0.015^{\mathrm{a}}$} \\
\hline III/IV & 39 & 10 & 29 & & \\
\hline \multicolumn{6}{|c|}{ Lymph node metastasis } \\
\hline Negative & 46 & 20 & 26 & \multirow[t]{2}{*}{0.474} & \multirow[t]{2}{*}{0.491} \\
\hline Positive & 44 & 16 & 28 & & \\
\hline
\end{tabular}

${ }^{\mathrm{a}} \mathrm{P}<0.05$.

results imply that Rab23 serves a role in CRC progression and prognosis.

Rab23 increases the expression of $\mathrm{Ki}-67$ and the proliferative ability of CRC cells. As shown in Fig. 2A, IHC staining demonstrated that tissues with high expression of Rab23 also exhibited a strong expression of $\mathrm{Ki}-67$, which is known as a classical proliferation marker. The positive correlation of Rab23 and Ki-67 was found to be statistically significant (Fig. 2B). To further confirm the effect of Rab23 on the expression of Ki-67, the expression of Rab23 in CRC cells was upregulated by plasmid transfection. Following the induction of Rab23 overexpression in SW1116 cells, the expression of Ki-67 was significantly increased at the mRNA (Fig. 2C) and protein (Fig. 2D) levels. In the CRC cell line HT29, overexpression of Rab23 also significantly increased the expression of Ki-67, both at the mRNA (Fig. 2E) and protein (Fig. 2F) levels. These findings suggested that Rab23 may have an effect on CRC proliferation. To further test this hypothesis, an MTT assay was performed, and the result revealed that overexpression of Rab23 significantly increased the proliferative ability of SW1116 (Fig. 2G) and HT29 (Fig. 2H) cells. These results demonstrated that Rab23 can significantly increase the proliferation of CRC cells.

ERK and AKT signaling pathways are required for Rab23 to regulate the proliferation of $C R C$ cells. To investigate the potential molecular mechanism, possible molecular targets were detected. Subsequent to Rab23 overexpression in SW1116
(Fig. 3A) and HT29 (Fig. 3B) cells, the phosphorylation of ERK and AKT was activated, whereas no significant changes were detected in the total ERK and AKT levels. This implied that the ERK and AKT signaling pathways could be activated by Rab23 in CRC cells.

When SW1116 (Fig. 3C) and HT29 (Fig. 3D) cells were incubated with the ERK inhibitor U0126 or the AKT inhibitor LY294002, the increase in Ki-67 expression induced by Rab23 overexpression was reduced. Furthermore, the MTT assay revealed that incubation with U0126 or LY294002 inhibited the proliferative abilities induced by Rab23 overexpression in the SW1116 (Fig. 3E) and HT29 (Fig. 3F) cells. Taken together, these results proved that Rab23 was able to increase the proliferation of CRC cells via the ERK and AKT signaling pathways.

\section{Discussion}

Rab23 is a member of the Ras-related small GTPase family, which serves a key role in the regulation of the Shh signaling pathway. High expression of Rab23 has been reported in several types of tumors. For instance, Wang et al (16) reported that Rab23 was overexpressed in human astrocytoma, and promoted cell migration and invasion through the regulation of Rac1. Liu et al (17) also reported that Rab23 was overexpressed and activated in hepatocellular carcinoma. In the present study, high expression of Rab23 was detected in CRC tissues using IHC analysis, and this 
A

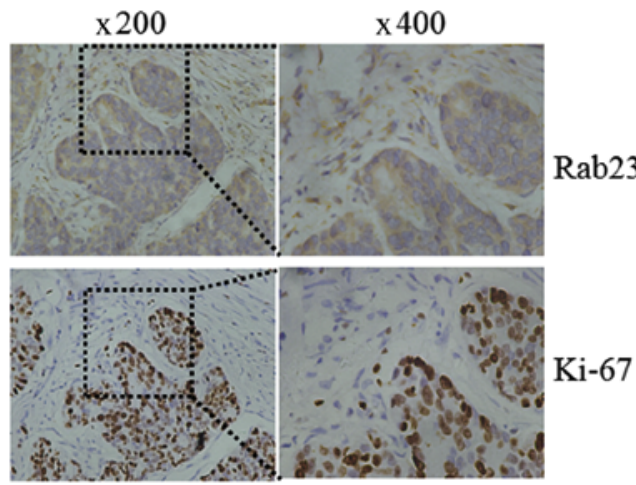

B

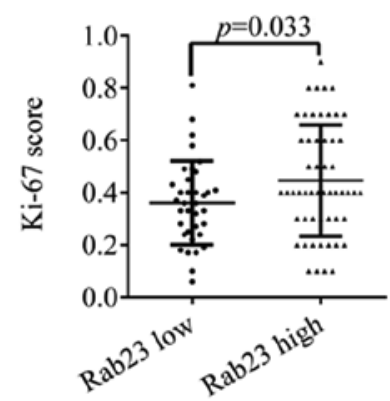

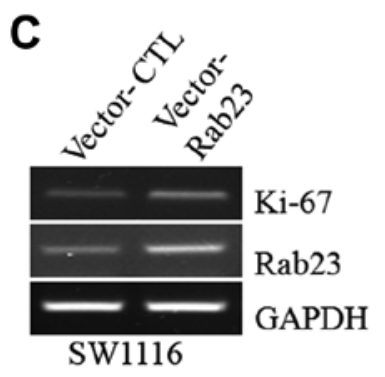

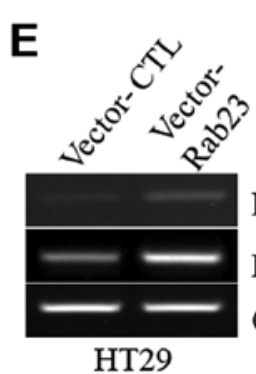

Ki-67

Rab23

GAPDH$$
\text { H129 }
$$

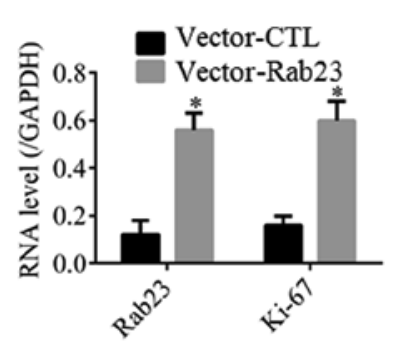

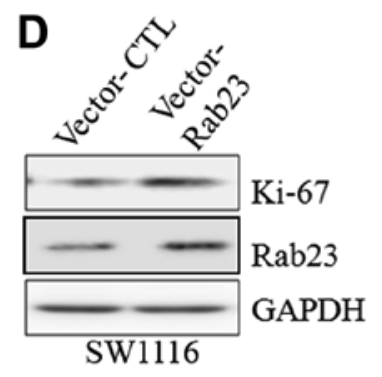
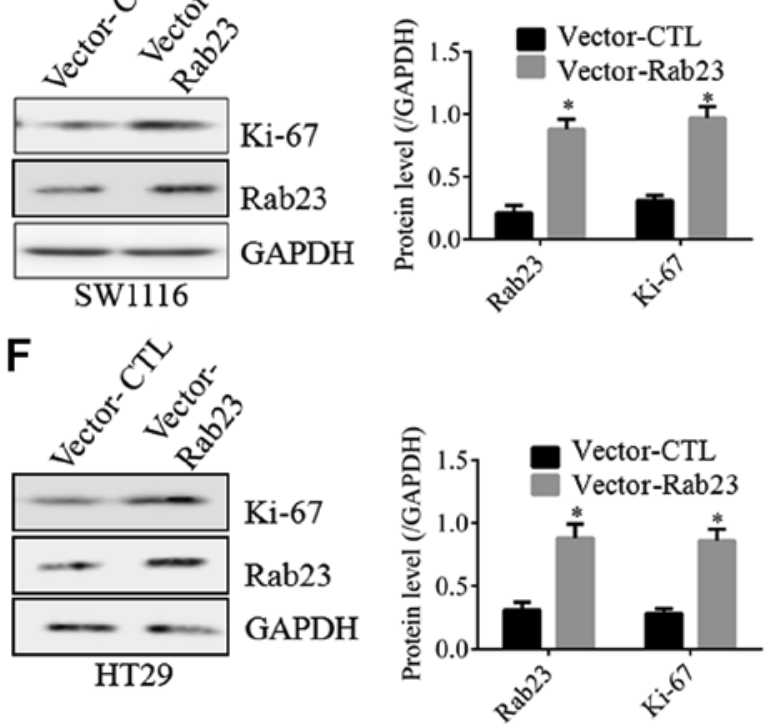
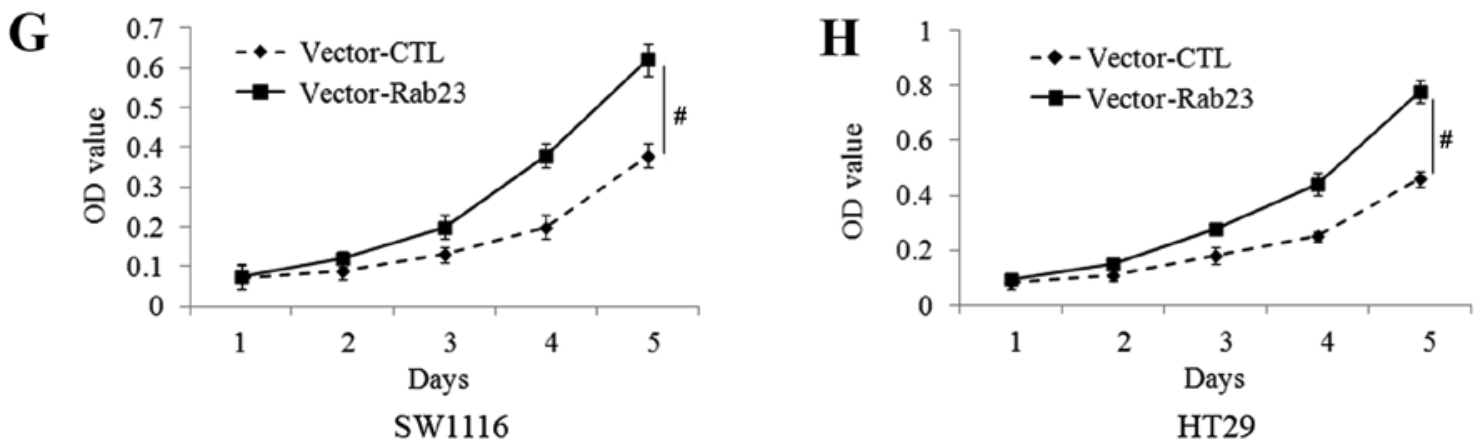

Figure 2. Rab23 increased the expression of Ki-67 and proliferation ability of CRC cells. (A) Immunohistochemical staining of Rab23 and Ki-67 in serial sections of CRC tissues, and (B) Ki-67 score showing a positive correlation with Rab23 expression. (C) mRNA and (D) protein expression levels of Rab23 and Ki-67 in SW1116 cells, examined by reverse transcription-polymerase chain reaction and western blot analysis, respectively. (E) mRNA and (F) protein expression levels of Rab23 and Ki-67 in HT29 cells. Rab23 overexpression in SW1116 and HT29 cells resulted in significantly increased expression of Ki-67 at the mRNA and protein levels. (G) SW1116 and (H) HT29 cell proliferation ability was increased following Rab23 overexpression. * P<0.05 vs. vector-CTL; ${ }^{\#} \mathrm{P}<0.05$. CRC, colorectal cancer; CTL, control; OD, optical density.

was further confirmed in CRC cells, both at the mRNA and protein levels. These results are consistent with the previously reported data by Wang et al (16) and Liu et al (17). Thus, it is suggested that Rab23 may serve as a diagnostic marker in CRC and may function as an oncogenic protein in CRC.

Thus far, the role of Rab23 has been reported in several types of tumors, but this remains under debate. Jiang et al (18) demonstrated that Rab23 promoted the proliferation and invasion of bladder cancer cells. In addition, Chang et al (19) found that Rab23 was required for tumor growth in prostate cancer. However, Liu et al (20) reported that ectopic expression of Rab23 inhibited the growth and proliferation, as well as induced cell apoptosis in breast cancer cells. In the present study, it was observed that high expression of Rab23 was positively associated with tumor size and advanced clinical stage. This implied an oncogenic role of Rab23 in CRC. Further cell experiments revealed that Rab23 promoted the expression of Ki-67 and the proliferative ability of CRC cells. The present study results are consistent with the findings of 


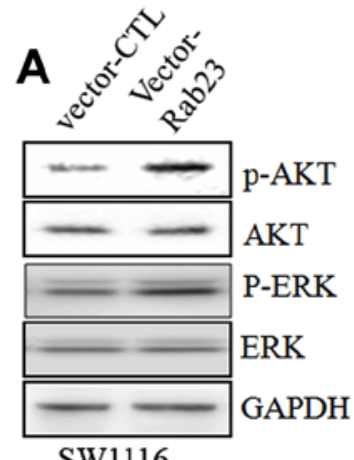

SW1116
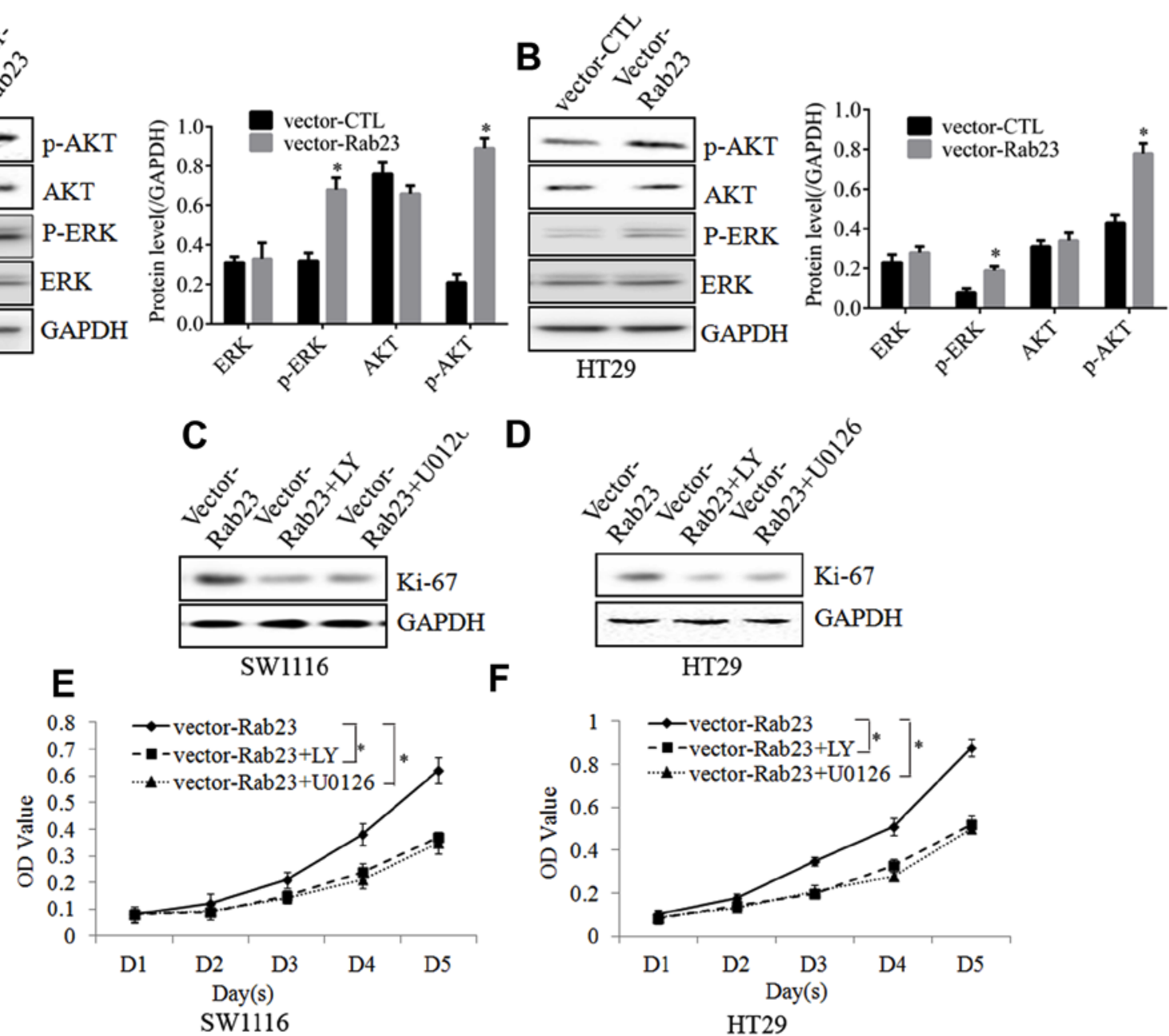

Figure 3. ERK and AKT signaling pathways are required for regulatory effect of Rab23 on colorectal cancer cell proliferation. Rab23 overexpression in (A) SW1116 and (B) HT29 cells resulted in increased phosphorylation of ERK and AKT. The expression of Ki-67 in (C) SW1116 and (D) HT29 cells was inhibited by addition of the ERK inhibitor U0126 or AKT inhibitor LY294002. (E) SW1116 and (F) HT29 cell proliferation ability was inhibited by addition of the ERK inhibitor U0126 or AKT inhibitor LY294002. "P<0.05 vs. Vector-Rab23. ERK, extracellular signal-regulated kinase; AKT, protein kinase B; CTL, control; OD, optical density; LY, AKT inhibitor LY294002.

Jiang et al (18) and Chang et al (19), although they differ from the results of the study conducted by Liu et al (20), and provide further evidence on the roles of Rab23 in the progression of CRC. Furthermore, the survival analysis performed in the current study demonstrated that Rab23 was positively associated with poor DFS. This is in agreement with the study by Zhang et al (13), which reported that Rab23 was positively associated with the poor prognosis of patients with ovarian cancer, implying that Rab23 may be used for predicting the prognosis of patients with CRC.

The ERK and phosphoinositide 3-kinase/AKT signaling pathways serve an important role in a multitude of cellular functions, including cell proliferation (21), survival (22), migration (23), apoptosis $(24,25)$ and angiogenesis (15). However, whether ERK or AKT can function as downstream targets of Rab23 remains unknown. In the present study, it was observed that overexpression of Rab23 activated the ERK and AKT signaling pathways. In addition, blocking the ERK or AKT signaling pathways eliminated the effects of Rab23 on the proliferation of CRC cells. This proved the important role of the ERK and AKT signaling pathways in the effect of Rab23 in CRC cell proliferation.
In conclusion, the present study confirmed the important role of Rab23 in CRC progression and poor prognosis, suggesting the potential use of Rab23 in diagnosis and prognosis prediction in this disease. The current study data provided a preliminary experimental basis for further research on Rab23 in CRC, and also suggested that Rab23 may serve as a novel treatment target for CRC.

\section{Acknowledgements}

The authors would like to thank all the members of the Pathology Department for their help in collecting tissue specimens, IHC staining and scoring.

\section{Funding}

No funding was received.

\section{Availability of data and materials}

The datasets used and/or analyzed during the current study are available from the corresponding author on reasonable request. 


\section{Authors' contributions}

HM designed this study and wrote the manuscript. TZ and DH collected the tissue specimens, performed IHC staining and analyzed the data.

\section{Ethics approval and consent to participate}

This retrospective study involving tissues from human patients was approved by the Ethics Committee of Shanxian Central Hospital.

\section{Patient consent for publication}

No applicable.

\section{Competing interests}

The authors declare that they have no competing interests.

\section{References}

1. Schreckenbach T, Zeller MV, El Youzouri H, Bechstein WO and Woeste G: Identification of factors predictive of postoperative morbidity and short-term mortality in older patients after colorectal carcinoma resection: A single-center retrospective study. J Geriatr Oncol 9: 649-658, 2018.

2. Issa AM, Hutchinson JF, Tufail W, Fletcher E, Ajike R and Tenorio J: Provision of personalized genomic diagnostic technologies for breast and colorectal cancer: An analysis of patient needs, expectations and priorities. Per Med 8: 401-411, 2011.

3. Zhen Y, Luo $\mathrm{C}$ and Zhang $\mathrm{H}$ : Early detection of ulcerative colitis-associated colorectal cancer. Gastroenterol Rep (Oxf) 6 : 83-92, 2018.

4. Mansfield C, Ekwueme DU, Tangka FKL, Brown DS, Smith JL, Guy GP Jr, Li C and Hauber B: Colorectal cancer screening: Preferences, past behavior, and future intentions. Patient 11: 599-611, 2018.

5. Satake H, Sunakawa Y, Miyamoto Y, Nakamura M, Nakayama H, Shiozawa M, Makiyama A, Kobayashi K, Kubota Y,Mori M, et al: A phase II trial of 1st-line modified-FOLFOXIRI plus bevacizumab treatment for metastatic colorectal cancer harboring RAS mutation: JACCRO CC-11. Oncotarget 9: 18811-18820, 2018.

6. Ihnát P, Vávra P, Slívová I, Tulinský L and Penka I: Radiotherapy in the treatment of rectal cancer is it time to move on? Rozhl Chir 97: 156-160, 2018 (In Czech).

7. Hathout L, Maloney-Patel N, Malhotra U, Wang SJ, Chokhavatia S, Dalal I, Poplin E and Jabbour SK: Management of locally advanced rectal cancer in the elderly: A critical review and algorithm. J Gastrointest Oncol 9: 363-376, 2018.

8. Olkkonen VM, Peterson JR, Dupree P, Lütcke A, Zerial M and Simons K: Isolation of a mouse cDNA encoding Rab23, a small novel GTPase expressed predominantly in the brain. Gene 138: 207-211, 1994.

9. Evans TM, Ferguson C, Wainwright BJ, Parton RG and Wicking C: Rab23, a negative regulator of hedgehog signaling, localizes to the plasma membrane and the endocytic pathway. Traffic 4: 869-884, 2003.
10. Guo A, Wang T, Ng EL, Aulia S, Chong KH, Teng FY, Wang Y and Tang BL: Open brain gene product Rab23: Expression pattern in the adult mouse brain and functional characterization. J Neurosci Res 83: 1118-127, 2006.

11. Eggenschwiler JT, Espinoza E and Anderson KV: Rab23 is an essential negative regulator of the mouse Sonic hedgehog signalling pathway. Nature 412: 194-198, 2001.

12. Jian Q, Miao Y, Tang L, Huang M, Yang Y, Ba W, Liu Y, Chi S and Li C: Rab23 promotes squamous cell carcinoma cell migration and invasion via integrin $\beta 1 /$ Rac1 pathway. Oncotarget 7 : 5342-5352, 2016

13. Zhang W, Yu F, Wang Y, Zhang Y, Meng L and Chi Y: Rab23 promotes the cisplatin resistance of ovarian cancer via the Shh-Gli-ABCG2 signaling pathway. Oncol Lett 15: 5155-5160, 2018.

14. Hari DM, Leung AM, Lee JH, Sim MS, Vuong B, Chiu CG and Bilchik AJ: AJCC cancer staging manual 7th edition criteria for colon cancer: Do the complex modifications improve prognostic assessment? J Am Coll Surg 217: 181-90, 2013.

15. Zeng $\mathrm{C}$, Wen $\mathrm{M}$ and Liu X: Fibroblast activation protein in osteosarcoma cells promotes angiogenesis via AKT and ERK signaling pathways. Oncol Lett 15: 6029-6035, 2018.

16. Wang M, Dong Q and Wang Y: Rab23 is overexpressed in human astrocytoma and promotes cell migration and invasion through regulation of Rac1. Tumour Biol 37: 11049-11055, 2016.

17. Liu YJ, Wang Q, Li W, Huang XH, Zhen MC, Huang SH, Chen LZ, Xue L and Zhang HW: Rab23 is a potential biological target for treating hepatocellular carcinoma. World J Gastroenterol 13: 1010-1017, 2007.

18. Jiang Y, Han Y, Sun C, Han C, Han N, Zhi W and Qiao Q: Rab23 is overexpressed in human bladder cancer and promotes cancer cell proliferation and invasion. Tumour Biol 37: 8131-8138, 2016.

19. Chang J, Xu W, Liu G, Du X and Li X: Downregulation of Rab23 in prostate cancer inhibits tumor growth in vitro and in vivo. Oncol Res 25: 241-248, 2017.

20. Liu Y, Zeng C, Bao N, Zhao J, Hu Y, Li C and Chi S: Effect of Rab23 on the proliferation and apoptosis in breast cancer. Oncol Rep 34: 1835-1844, 2015.

21. Xu J, Pan X and Hu Z: MiR-502 mediates esophageal cancer cell TE1 proliferation by promoting AKT phosphorylation. Biochem Biophys Res Commun 501: 119-123, 2018.

22. Zohrap N, Saatci Ö, Ozes B, Coban I, Atay HM, Battaloglu E, Şahin Ö and Bugra K: SIK 2 attenuates proliferation and survival of breast cancer cells with simultaneous perturbation of MAPK and PI3K/Akt pathways. Oncotarget 9: 21876-21892, 2018.

23. Yao WF, Liu JW and Huang DS: MiR-200a inhibits cell proliferation and EMT by down-regulating the ASPH expression levels and affecting ERK and PI3K/Akt pathways in human hepatoma cells. Am J Transl Res 10: 1117-1130, 2018.

24. Qi Z, Yin L, Xu Y and Wang F: Pegylated liposomal-paclitaxel induces ovarian cancer cell apoptosis via TNF-induced ERK/AKT signaling pathway. Mol Med Rep 17: 7497-504, 2018.

25. Park CH, Han SE, Nam-Goong IS, Kim YI and Kim ES: Combined effects of baicalein and docetaxel on apoptosis in $8505 \mathrm{c}$ anaplastic thyroid cancer cells via downregulation of the ERK and Akt/mTOR pathways. Endocrinol Metab (Seoul) 33: 121-132, 2018.

This work is licensed under a Creative Commons Attribution-NonCommercial-NoDerivatives 4.0 International (CC BY-NC-ND 4.0) License. 